\title{
Design and Implementation for 3-DoF SCARA Robot based PLC
}

\author{
Iman Salih Karem* Talal A. Jabbar A.Wahab** \\ Mawadah Jlaa Yahyh**** \\ *,**,***Department of Electromechanical Engineering /University of Technology \\ *Email: dr_esk71@yahoo.com \\ ***Email:electromechanical19@yahoo.com
}

(Received 21 August 2016; accepted 22 January 2017)

https://doi.org/10.22153/kej.2017.01.002

\begin{abstract}
This paper presents mechanical and electrical design, and implementation process of industrial robot, 3-DoF type SCARA (selective compliment assembly robot arm), with two rotations and one translation used for welding applications. The design process also included the controller design which was based on PLC(programmable logic controller) as well as selection of mechanical and electrical components. The challenge was to use the available components in Iraq with reasonable costs. The robot mentioned is fully automated using programmable logic controller PLC(Zelio type SR3-B261BD), with 16inputs and 10 outputs. The PLC was implemented in FBD logic to obtain three different automatic motions with high efficiency withouterror,where the two links can stop in a certain points and link 3 bushed down for doing the task in a desired trajectory motion by programming the PLCcontroller.
\end{abstract}

Keywords: DoF, FBD, PLC, SCARArobot.

\section{Introduction}

Applying autonomy is a comparatively youthful domain of current technology that crosses conventional engineering limits. Comprehension the multifaceted nature of robots and their implementations requirements learning of electrical engineering, mechanical designing, frameworks, modern building, software engineering, mathematics and economics.

The robot is a reprogrammable multifunctional controller designed to handling a material, accessories, devices, or particular gadgets through variable programmed motions for the fulfillment for a set of assignments [1]. The robots display significantly convoluted electromechanical systems with common collaborations of robot mechanics and drives, at configuration of which the mechatronic methodology ought to be taken into consideration [2].
An industrial robot is a programmable, multipractical controller designed to remove materials, parts, apparatuses, or special gadgets through variable modified movements for the fulfillment of a set of tasks [3].

PLC is simply a special computer device used for industrial control systems. That had an input and an output interface, controlled by a simulation program designed by a computer; it is used for for electromechanical automated process.

This contains major components processor unit, power supply, programming device, program and data memory, input and output interface and communications interface as shown in figure(1). [4] 


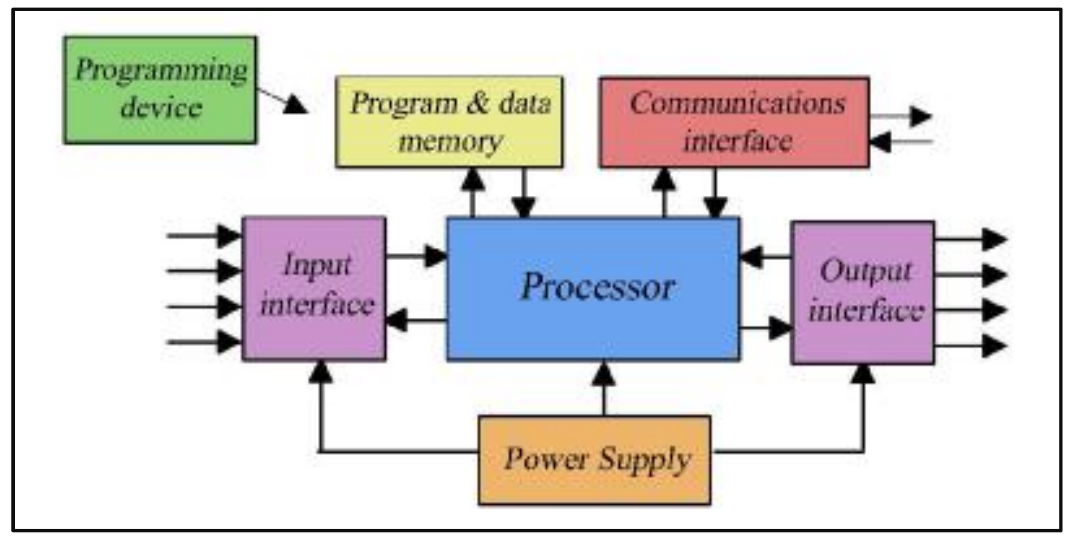

Fig. 1. The PLC system [4].

There are several literatures are available to present research, Pouyaand Kourosh,(2012) [5] presented, hypotheticl prototype model, simulated and optimized of a 3 DOF industrial SCARA robot with PID controller after optimization with Genetic algorithm. Mohammad and Mahdi ,(2012) [6]a trditional PD controller was used then a comparison method with a neural network controller system to reach the exact position control and motion characteristics of SCARA arm. Firas and Ahmed, (2013) [7] proposed a control system to the four degree of freedom (4DOF) Feedback Instruments IVAX SCARA robot manipulator using field programmable gate array(FPGA) kit to implemented the inverse kinematic calculations processing. Jian and Wei, (2013) [8] studied Four degrees of freedom SCARA robot kinematics modeling, using the Robotics Toolbox forward kinematics of the robot inverse kinematics simulation in the MATLAB environment. Through simulation the observed movement of each joint SCARA robotic state verification the proposed modeling was corrected to reach the desirable goal by enter the desirable joint angles, and the system output end of the robotic is to reach the space coordinates. Sudhaet al., (2013) [9] concentrated on the construction of a Robotic arm or a manipulator with SCARA configuration. The manipulator consists of two revolute joints are rotating joints which create rotation about their axis fitted with two DC Motor are interfaced with the ATMEGA 16 Microcontroller board using a L293D IC, and one prismatic joint. Yousif, (2013) [10] focused in this research on The SCARA Robot which had four degrees of freedom (DOFs), with three (shoulder, elbow, wrist) controlling by servo motors and one by pneumatics. It is realized by a 3D hypothetical reality (VR) modeling, which builds and receives instruction through a MATLAB/Simulink in Version R2012a, develops an ANFIS (Adaptive Neuro Fuzzy Inference Strategy) control scheme for SCARA robot parameters. Zelun et al., (2013) [11] proposed the design of a hardware structure and software of 3-DOF industrial spot welding robot, The robot controlled by the PLC. Panchanand and Biswal, (2014) [12] presented a structure of artificial neural network (ANN) pattern to find the inverse kinematics solution of a 4- DOF SCARA arm. Morteza et al., (2014) [13] presented mechanical design of an educational manipulator of industrial SCARA robot, called FUMSCARA, The designing process contianed, designing of joint, link design, PID controller design and chosen of mechanical and electrical hardware, a critical trajectory in robot's workspace is traced. Nitesh et al., (2014) [14] Presented dynamic model of 3-DOF SCARA robot manipulator based on Lagrange Euler formulation using Mamdani based Fuzzy controller overcome the drawbacks of conventional PID controller. Subhashini et al., (2014) [15] presented SACRA robot model and the simulation used Unigraphics CAD software for deburring operation of circular elements. A complete mathematical modeling was pregnancies out for kinematics and equations are derived using the Denavit-Hartenberg notation.

In this paper a designing process and implementation of an industrial robotic arm of 3DoF type SCARA (Selective Compliant Assembly Robot Arm) with two rotations and one translation link used for welding applications. The robot mentioned is fully automated using programmable logic controller PLC based FBD language to obtain three different automatic motions. 


\section{Robot Kinematics}

The SCARA robot has three DOF.A schematic diagram assigning all the joint axes is represented in figure (2), joint 1 and joint 2 are rotations about $\mathrm{z}$-axis and joint 3is translation vertical.The three DOF manipulator kinematic parameters are derived using Denavit- Hartemberg formulation shown in Table (1)

Table 1,

Denavit-Hartenberg Parameters .

\begin{tabular}{lllll}
\hline Axis No. & Twist angles & Link length & Link offset & Joint angles \\
$\mathrm{i}$ & $\alpha_{\mathrm{i}-1}$ & $\mathrm{a}_{\mathrm{i}-1}$ & $\mathrm{~d}_{\mathrm{i}}$ & $\theta_{\mathrm{i}}$ \\
1 & 0 & 0 & & $d \theta_{1}$ \\
2 & 0 & $L$ & 0 & $\theta_{2}$ \\
3 & 0 & & $L$ & 0 \\
\hline
\end{tabular}

The forward kinematics include all values of the link transformations that can be multiplied together to find the single transformation from the base, Frame (0) to the tip of end- effector frame (3).

$$
\begin{aligned}
& \text { end-effector } T={ }_{3}^{0} T={ }_{1}^{0} T{ }_{2}^{1} T{ }_{3}^{2} T \\
& { }_{3}^{0} T=\left[\begin{array}{cccc}
r_{11} & r_{12} & r_{13} & p_{x} \\
r_{21} & r_{22} & r_{23} & p_{y} \\
r_{31} & r_{32} & r_{33} & p_{z} \\
0 & 0 & 0 & 1
\end{array}\right] \\
& {\left[\begin{array}{cccc}
c_{1} & -s_{1} & 0 & 0 \\
s_{1} & c_{1} & 0 & 0 \\
0 & 0 & 1 & d_{1} \\
0 & 0 & 0 & 1
\end{array}\right] *\left[\begin{array}{cccc}
c_{2} & -s_{2} & 0 & L_{1} \\
s_{2} & c_{2} & 1 & 0 \\
0 & 0 & 0 & 0 \\
0 & 0 & 0 & 1
\end{array}\right] *} \\
& {\left[\begin{array}{cccc}
1 & 0 & 0 & L_{2} \\
0 & 1 & 0 & 0 \\
0 & 0 & 1 & -D_{3} \\
0 & 0 & 0 & 1
\end{array}\right]} \\
& =\left[\begin{array}{cccc}
c_{12}-s_{12} & -s_{2} c_{1}-s_{1} c_{2} & 0 & c_{1} L_{1} \\
s_{1} c_{2}+c_{1} s_{2} & -s_{12}+c_{12} & 0 & s_{1} L_{1} \\
0 & 0 & 1 & d_{1} \\
0 & 0 & 0 & 1
\end{array}\right] * \\
& {\left[\begin{array}{cccc}
1 & 0 & 0 & L_{2} \\
0 & 1 & 0 & 0 \\
0 & 0 & 1 & -D_{3} \\
0 & 0 & 0 & 1
\end{array}\right]} \\
& =\left[\begin{array}{cccc}
c_{12} & -s_{12} & 0 & c_{12} L_{2}+c_{1} L_{1} \\
s_{12} & c_{12} & 0 & s_{12} L_{2}+s_{1} L_{1} \\
0 & 0 & 1 & -D_{3}+d_{1} \\
0 & 0 & 0 & 1
\end{array}\right] \\
& r_{11}=c_{12}, r_{21}=s_{12}, r_{31}=0, r_{12}=-s_{12}, r_{22}=c_{12}, r_{32}= \\
& 0, r_{13}=0, r_{23}=0, r_{33}=1 \\
& p_{x}=c_{12} L_{2}+c_{1} L_{1} \\
& p_{y}=s_{12} L_{2}+s_{1} L_{1} \\
& p_{z}=d_{1}-D_{3}
\end{aligned}
$$

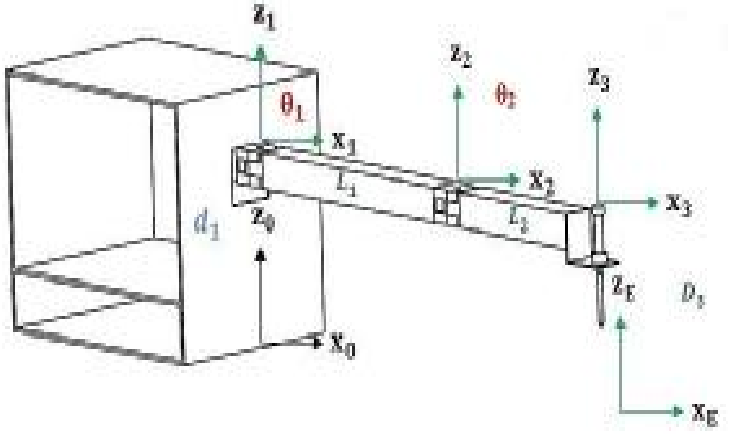

Fig. 2. Frames of Manipulator.
In this papers, Algebraic solution approach was used for deriving the inverse kinematics solution that is to determine the value of angle $(\theta 1),(\theta 2)$ and distance (D3)as in[16].

$$
\begin{aligned}
& \theta_{2}=A \quad \tan 2\left(\sqrt{1-\left(\frac{\mathrm{p}_{\mathrm{x}}{ }^{2}+\mathrm{p}_{\mathrm{y}}{ }^{2}-\mathrm{L}_{1}{ }^{2}-\mathrm{L}_{2}{ }^{2}}{2 \mathrm{~L}_{1} \mathrm{~L}_{2}}\right)^{2}},\right. \\
& \left.\frac{\mathrm{p}_{\mathrm{x}}{ }^{2}+\mathrm{p}_{\mathrm{y}}{ }^{2}-\mathrm{L}_{1}{ }^{2}-\mathrm{L}_{2}{ }^{2}}{2 \mathrm{~L}_{1} \mathrm{~L}_{2}}\right)
\end{aligned}
$$

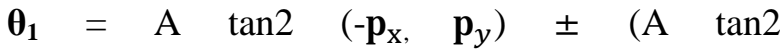

$$
\begin{aligned}
& \left(\sqrt{p_{\mathrm{x}}{ }^{2}+\mathrm{p}_{\mathrm{y}}{ }^{2}-\left(\mathrm{s}_{2} \mathrm{~L}_{2}\right)^{2}}, \mathrm{~s}_{2} \mathrm{~L}_{2}\right) \\
& D_{3}=d_{1}-p_{z}
\end{aligned}
$$

We can find the linear and angular velocity of end-effector by computing the basic Jacobian[16].

$$
\begin{aligned}
& {\left[\begin{array}{l}
\vec{v} \\
\vec{\omega}
\end{array}\right]=[J(\theta)] \cdot\left[\begin{array}{c}
\dot{\theta_{1}} \\
\dot{\theta} \\
\vdots \\
\vdots \\
\theta_{n}
\end{array}\right] \Longrightarrow\left[\begin{array}{c}
\dot{\theta_{1}} \\
\dot{\theta_{2}} \\
\vdots \\
\vdots \\
\dot{\theta}_{n}
\end{array}\right]=} \\
& \left.J J^{-1}\right] \cdot\left[\begin{array}{c}
\vec{v} \\
\vec{\omega}
\end{array}\right], \\
& J(\theta)=\left[\begin{array}{ccc}
\mathrm{s}_{2} L_{1} & 0 & 0 \\
\mathrm{c}_{2} L_{1}+L_{2} & L_{2} & 0 \\
0 & 0 & -1 \\
0 & 0 & 0 \\
0 & 0 & 0 \\
1 & 1 & 0
\end{array}\right]
\end{aligned}
$$

\section{SCARA Robot Design Process}

Scara manipulator is a nonlinear and time variant system, the applied controllers are necessary to have variation capability where linear models are inadequate to complete high 
accuracy and various performance operations [17].

To fulfill research aims are divided the design process into mechanical and electrical parts.

\subsection{Design of Mechanical Parts}

The initial concept of robotic design included the mechanical equipments. This section explain the construction of each part in the SCARA robot model .The test specification are given in appendix (A).

a. Links: Two Links as a manipulator arm square hollow section bar.The center to center distance of the link 1 is (470) $\mathrm{mm}$, and the center to center distance of the link 2 is (335) $\mathrm{mm}$, as shown in figure (3).

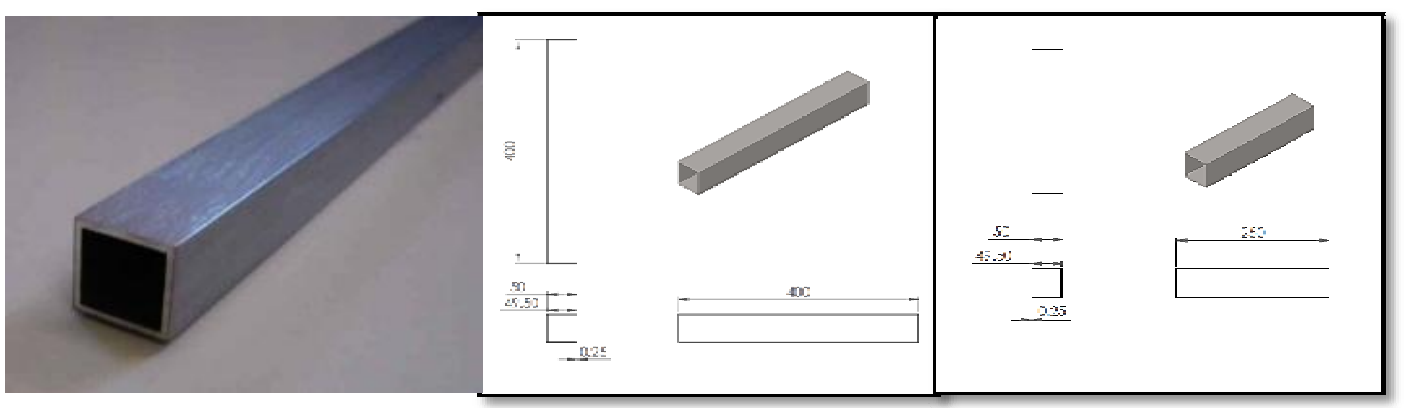

Fig. 3. Link Form Stainless Steel.

b. Joints: Two revolute joints square solid section, manufactured by milling machine and drilling it from the center to connect together to evaluate links as shown in figure (4).

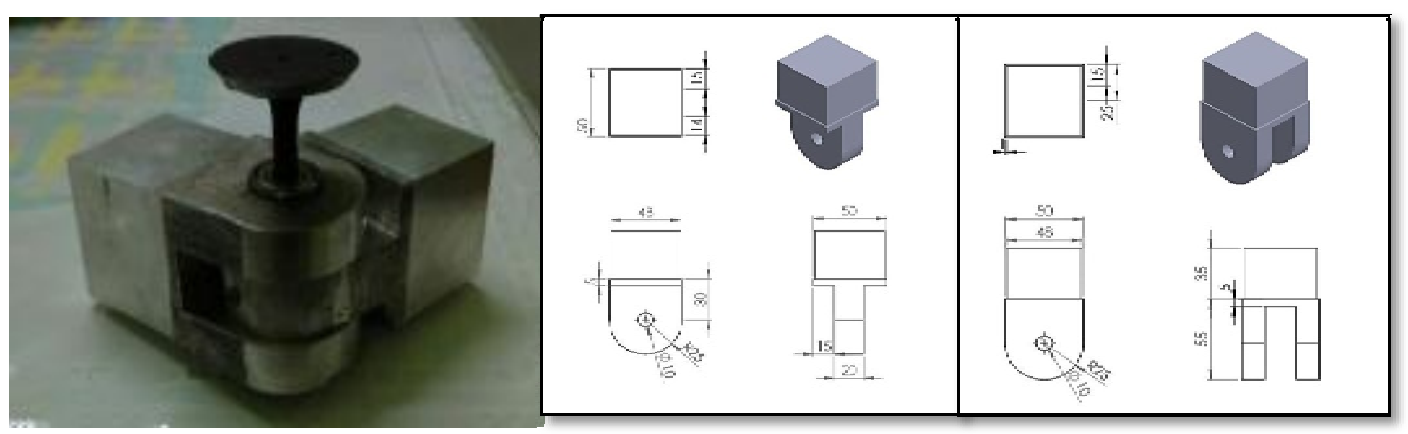

Fig. 4. Joint Connect The Arm.

c. Toothed Pulleys: The various three types are used in the current work. Vary in diameter, tooth shape, width of the gear and tooth sizes shown in figure (5).

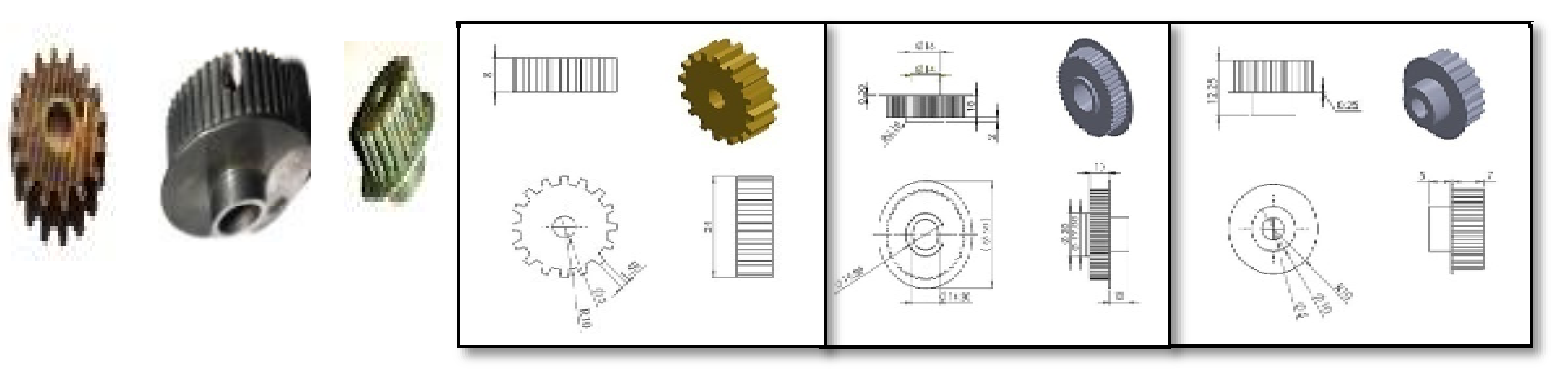

Fig. 5. The Toothed Pulleys.

d. Belt: Belt drives are widely used in many industries for power transmission since they are cheap and easy to maintain. The main purpose of belt drives is to transfer power between machines. 
e. Ball Bearing: The bearing is a machine element that constrains the relative motion to only the desired motion, and reduces friction between moving parts. Used 6200RS Red Rubber Sealed as shown in figure (6)

f. Tool Catch: An angle iron with a flat metal rod that has been folded to a 90-degree angle along its length, resulting in an L-shaped piece. Linkage with the end of the second link by bolt, to fixation the tool of different tasks as shown in figure (7).

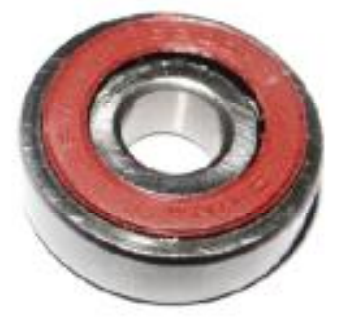

Fig. 6. Ball Bearing.
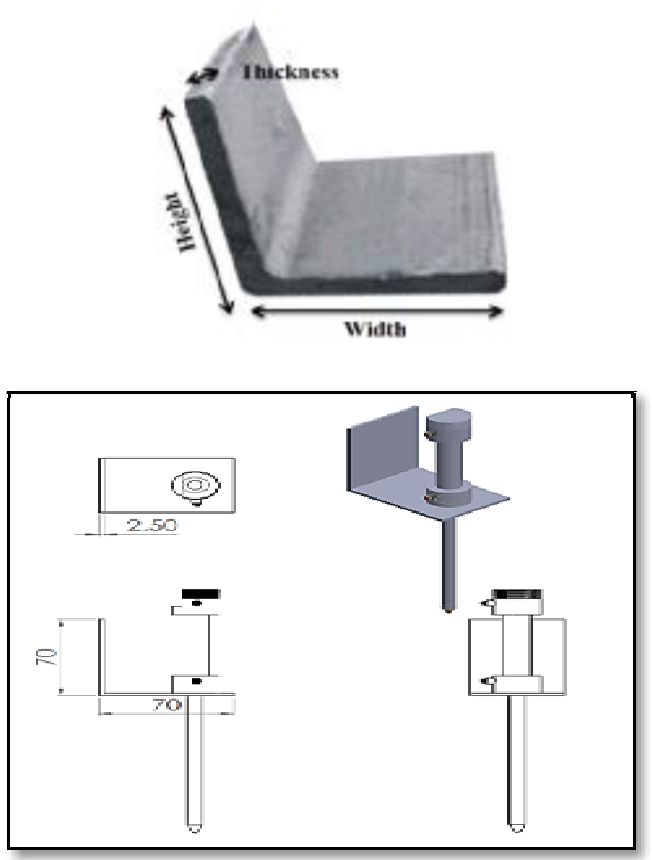

Fig. 7. Tool Catch.

g. Base Box Structure: Designed and implemented box to put and install the motors with photoelectric, and solenoid valve. The box covered by a plate of aluminum is shown in figure (8).

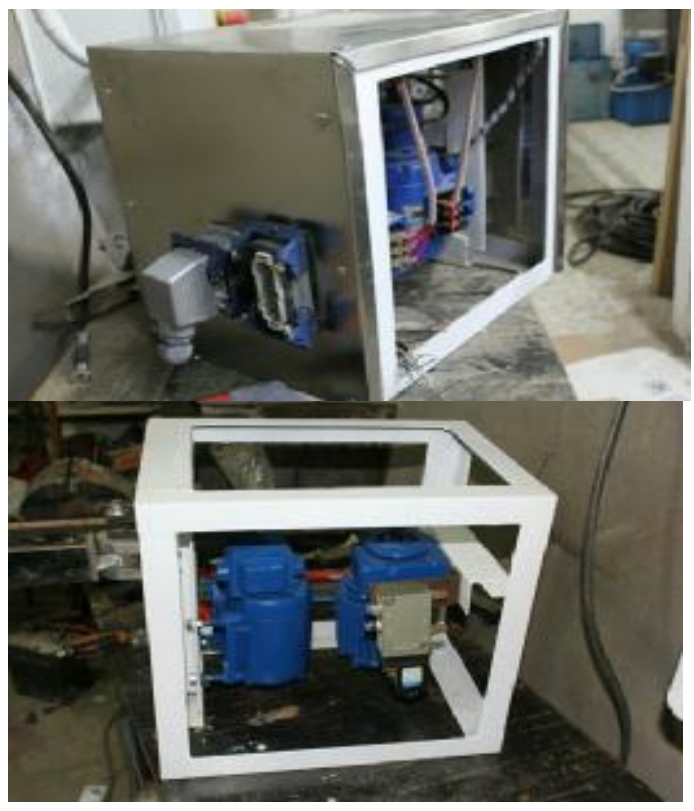

Fig. 8. Box From Iron and Covered by Aluminum.

\subsection{The Electrical Parts}

The electrical parts for operation the SCARA robot are included the following devices:

a. Photoelectric Sensor: Used photoelectric type Retro reflective and BEN5M-MFR model power supply $24-240 \mathrm{VAC} \pm 10 \% 50 / 60 \mathrm{~Hz}$ and infrared led source selectable light ON or Dark ON by switch.

b. Plugs and Sockets: Used two types (EBM10CF00)to connect between SCARA model and control panel.It can beseparated them to facilitate transmitted it.

c. Power Supply: A power supply model MDR100-24is an electronic device that supplies electric energy to an electrical load. Convert one form of electrical energy to another. A power input receives energy from the energy source, and a power output that transfers energy to the load.

d. Circuit breaker: Is one of the most important safety mechanisms, these simple machines cut the power when any problem occurs.

e. Relay: Relays are a remote control electrical switch that can be switched using low current to control a high current load and used in many applications, used type[ RXM4AB1BD] from Schneider Electric

f. Inverter: used to change the speed of electric motor called ac drive.It is used Type N700E operating by power 220 volt.

g. Solenoid: Consists of a coil of wire with an iron plunger that is allowed to move through 
the center of the coil. An electric current through the coil creates a magnetic field. The magnetic field exerts a force on the plunger.

h. Prismatic: Used standard cylinders from FESTO Type DSNU-20-2 with maximum pressure 10 bar made of stainless steel and the bearing and end caps made of wrought aluminum alloy.

i. Air Compressor:TheEinhell air compressor is a device that converts power into potential energy stored in compressed air that mean supplies the system with compressed air.

j. Motors: Used for driving the links of the robot. The torque of motors provides high starting torque and sloping characteristics. The two motors have 100 watt and $2800 \mathrm{rpm}$ were used in the current work.

k. Controlling Robotic Arm:Zelio has analog and discrete $\mathrm{I} / \mathrm{O}, 16$ input ports, which are can be set as 16 discrete inputs or 10 discrete inputs with 6 analog inputs and 10 ports of discrete output. PLC made in Schneider Electric type SR3-B261BD operating by power 24VDC. Connected to PC by serial port in order to use the software program( FBD language), the main advantage is that programs written in Function Block easy to follow solely follow the trajectory.

1. Cable Tray:Used the cable tray made from plastic PVC light weight, easy installation, corrosion resistance and channel slotted track.

\section{Control System Design Based PLC}

The software implementation of a SCARA Robot used FBD is a graphical language that lets users simply depict complex steps by easily linking together function blocks, like drawing a circuit diagram with the help of a graphical.[18] This language is similar to linking a diagram more than Ladder code. With FBD the blocks are "cabled" together sequently to follow easily. The same instructions as Ladder utilize, but visually is more understandable to a viewer than relay logic. The main advantage is that programs written in Function Block easy to follow solely follow the trajectory[19].

Used zelio software and FBD languages uses logic functions in the block diagrams to implement in the controller SCARA robot model.Figure(9) depicts this programming language, where the left side is the system input, and the right side is the system output.
Moreover checks all parts collecting together suitably and the control panel connecting wires correctly and safely as shown in figure (10). The detailed control system can be illustrated as the flowchart given in figure (11).

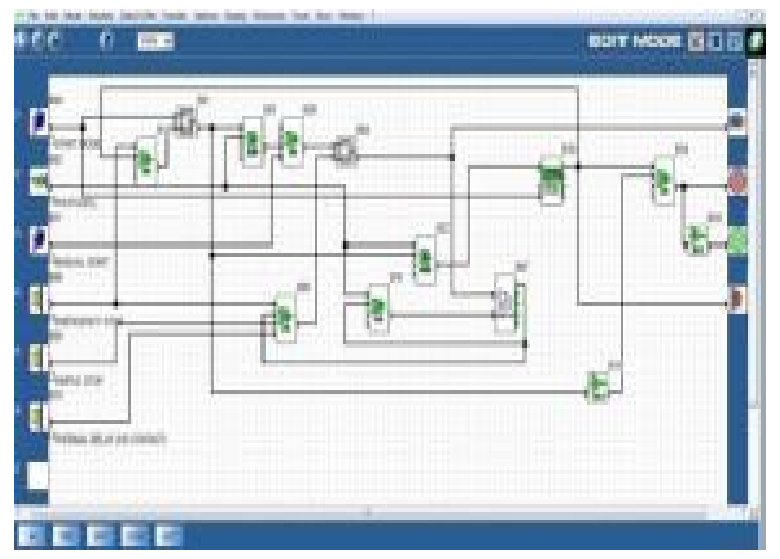

Fig. 9. The Programming of Zelio.

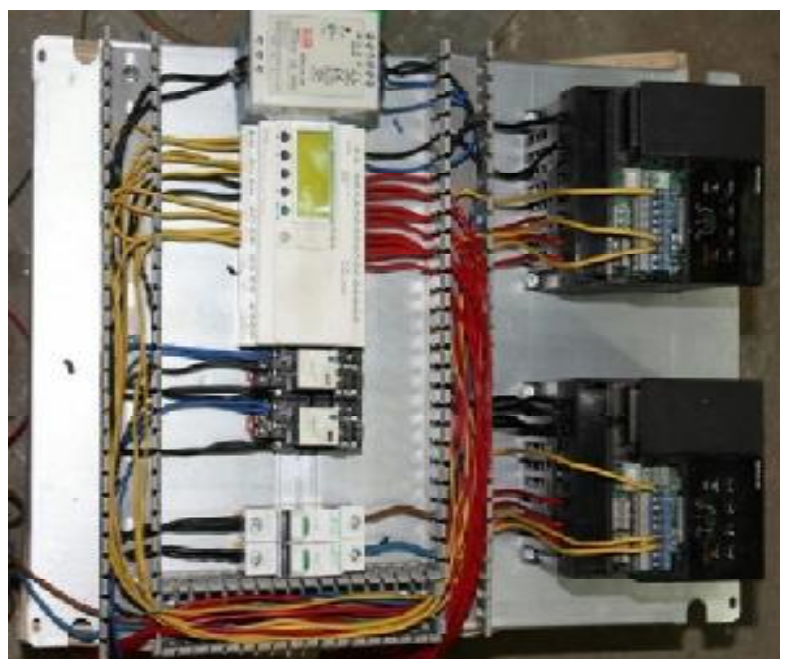

Fig. 10. Control Panal System for SCARA Robot

\section{Experimental Work}

The robot's mechanical and electrical parts are combined as in figure (12) to run and implement three experimental works to obtain three trajectory motions anti clock wise. The schematic block diagram for overall system illustrated in figure (13) The two links were stopped in certain points and link3 bushed down for doing the task in a desired trajectory motion. The performance of a robot was tested in test trajectory from the simulation results based on inverse kinematic equations to get theta valus using Matlab softwer for each experimental. 


\section{a. First Experimental}

The motion of the two links was programmed for the theta from $\theta_{1}=0$ to $\theta_{16}=150^{\circ}$ with the same time . 16 theta values were chosen in the 16 axes ,figure (14) depicts the desired first path from starting point to the destination point, by these set values, found that in softwares Matlab.A robot manipulator to move its trajectory at a constant velocity in this experimental $\theta=0^{\circ}-$ $150^{\circ}$ as identified in the program of PLC,see figure (15), each point(red points) describes the position of the end effector of the robot.The two links move in the same theta and make an arc like that in figure (14) .

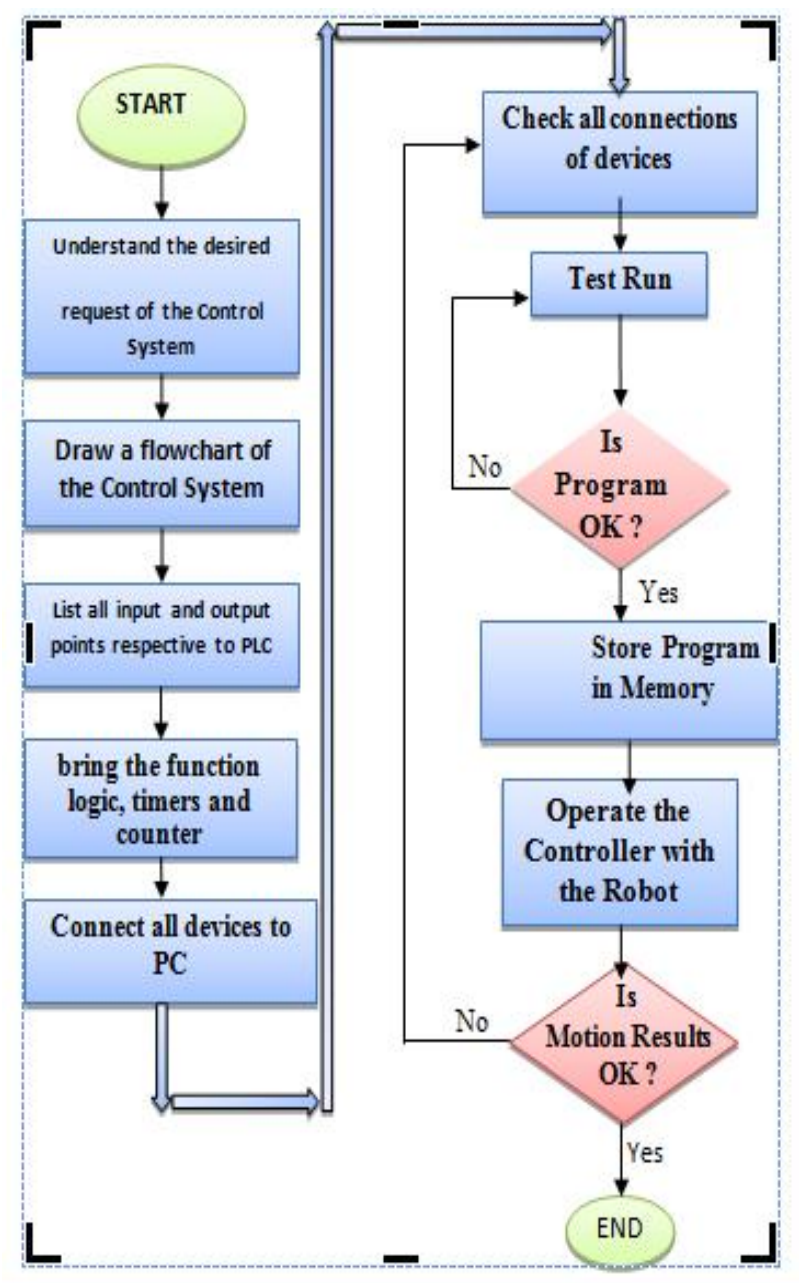

Fig. 11. Flowchart of controller design for SCARA robot.

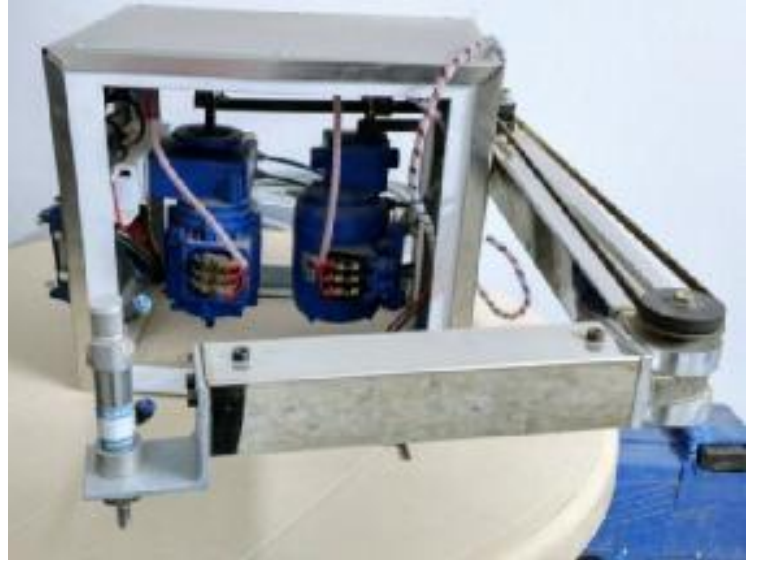

Fig. 12. Final SCARA Industrial Robot.

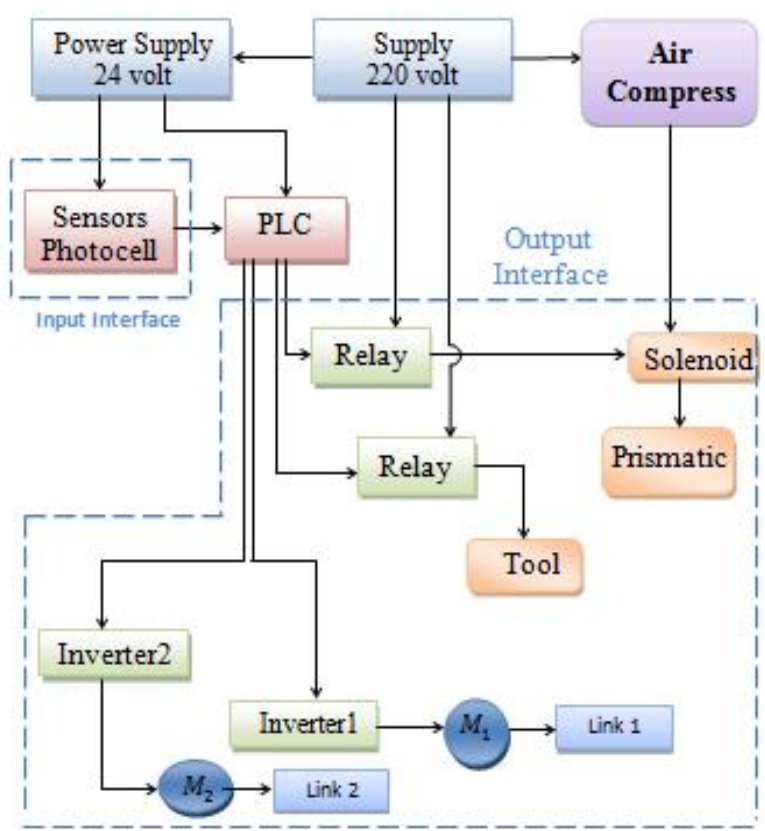

Fig. 13. The Schematic Block Diagram for Overall System.

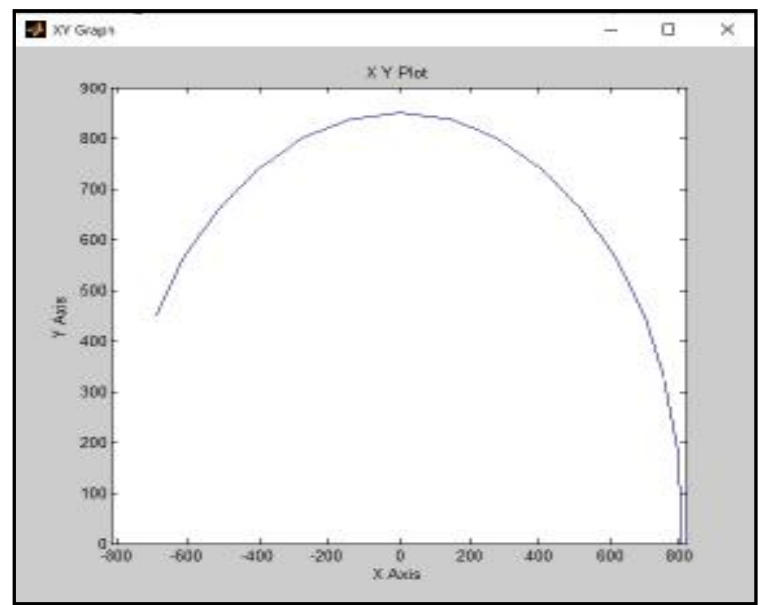

Fig. 14. Tthe Desired First Path. 


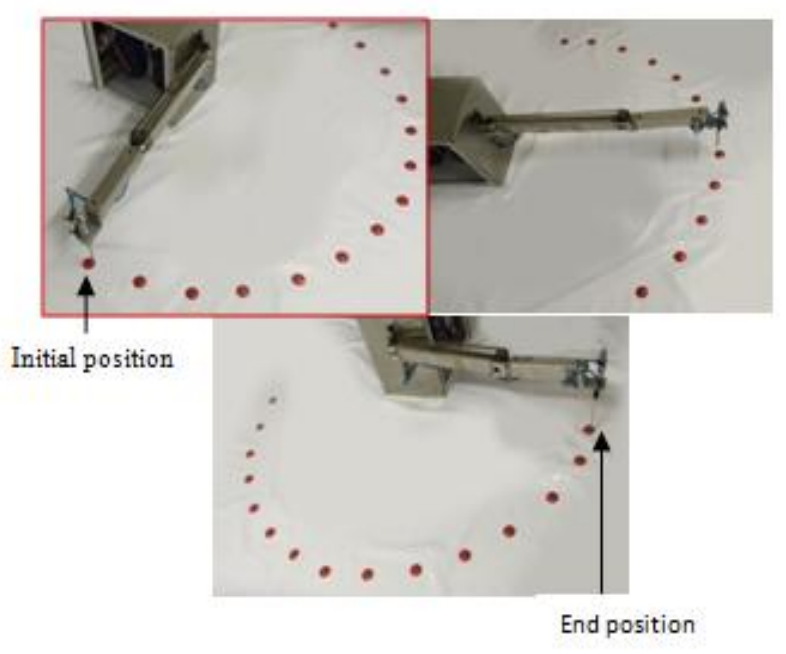

Fig. 15. The First Practical (Actual) Motion.

\section{b. Second Experimental}

Chose 11 theta values in the 11 axes to creat a disiered path as shown in the figure (16a).Practically the two links moved together from $0^{\circ}$ to $90^{\circ}$ the angle between them equal zero as shown in figure (16-b),then the link1 stopped just link 2 moves made an angle between Links increase 10 degree for each new position depended on controller program PLC. Each point describes the position of the end effector as shown in figure (16) .

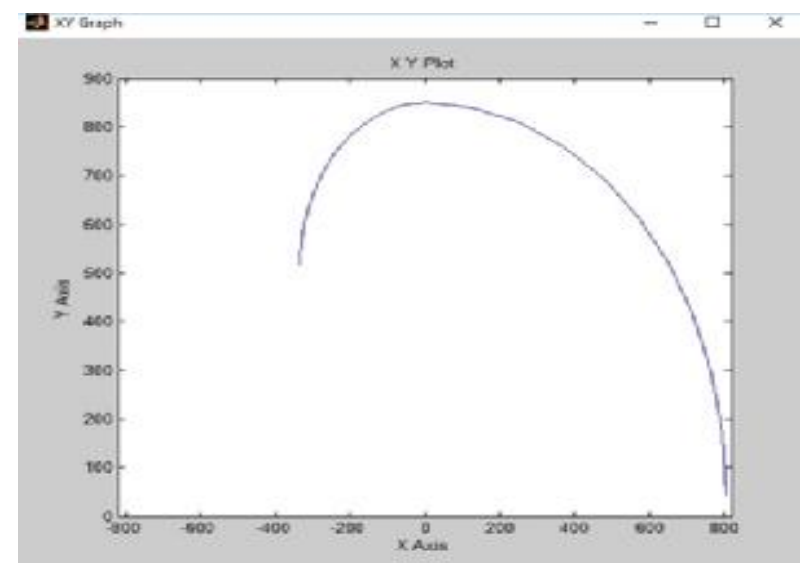

(a)

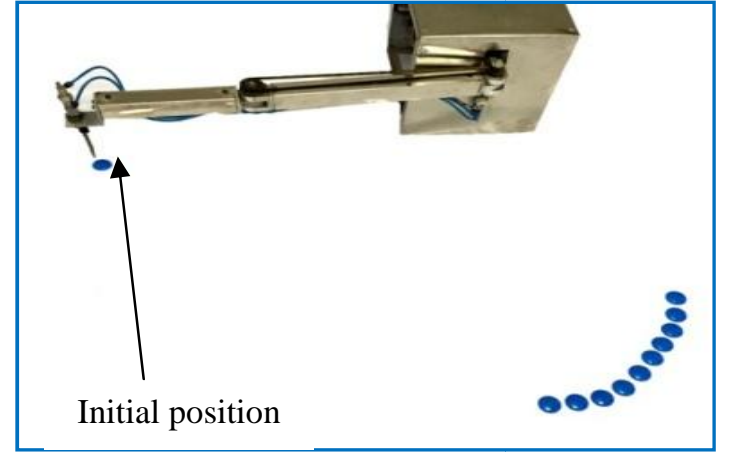

(b)

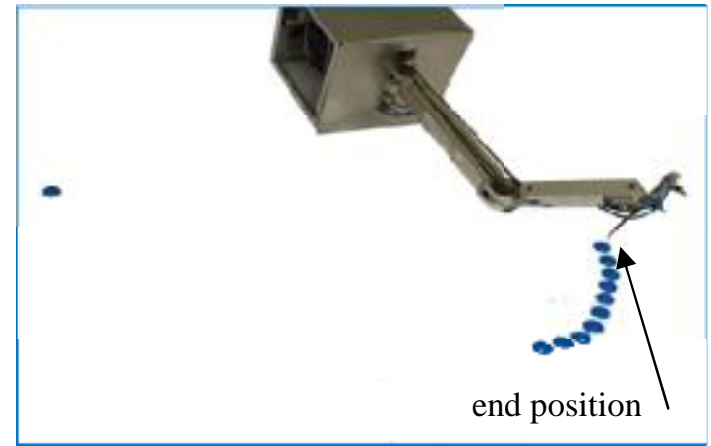

(c)

Fig. 16. ( a) the desired second path.(b\&c) The Second Practical Motion

\section{c. Third Experimental}

In this motion different 11 theta values $\theta_{1,2}=$ 0 to $\theta_{1,2}=50$ in the 11 axes were fed into Matlab softwere program to creat the desired path as shon in figure (17-a).Prracticaly identify the same value of theta in PLC program to obtain the trajectory actual motion, so the links moves together as shown in figure (17-b) after that the $\mathrm{L}_{1}$ movedand $\mathrm{L}_{2}$ stopped to a chave the trajectory motion as shown in the figure (17-c)

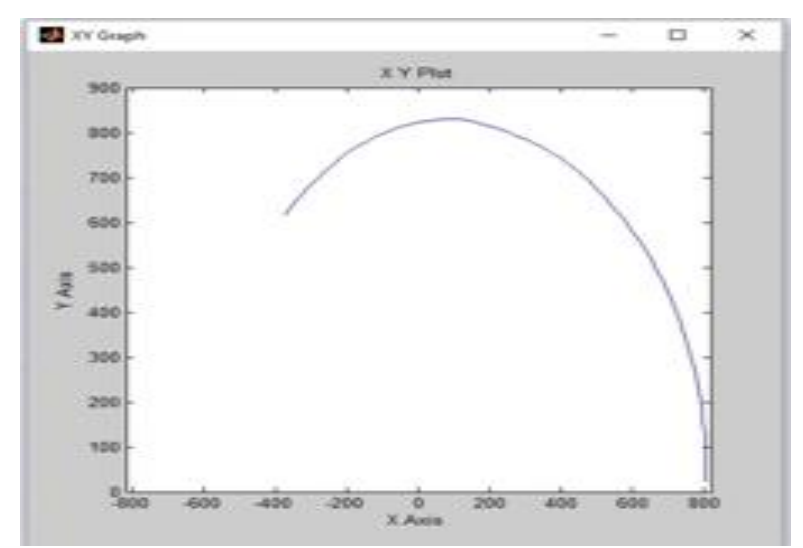

(a) 


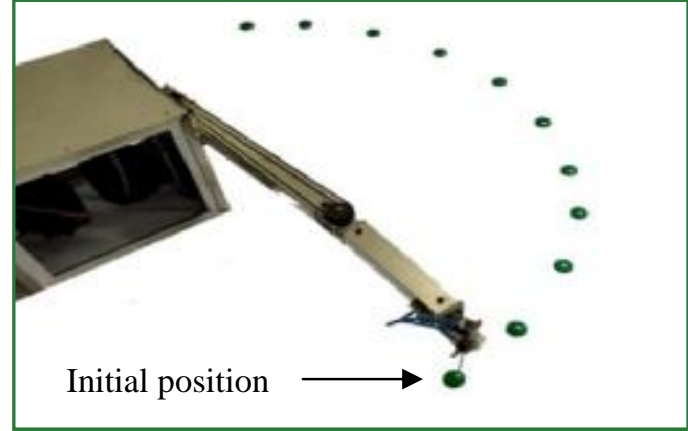

(b)

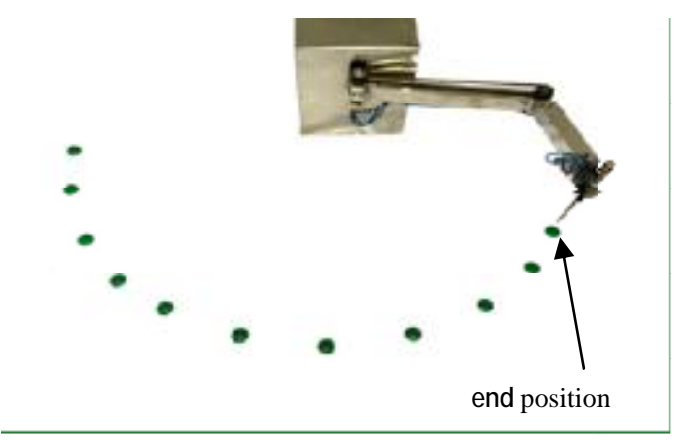

(c)

Fig. 17. (a) The Path for The Third Motion in Matlab (b\&c) The Third Practical Motion.

\section{Conclusions and Discussion}

In this paper,a special steps in design process are done for 3- DoF SCARA robotic arm. A three trajectory motion achieved firstly select the desired trajectory path based on the inverse kinematic analysis and using a softwere Matlab program to draw the path.

SCARA robot is a nonlinear and time invariant system .To overcome the difficulty and limitation of traditional controller,the PLC is applied which has running test before connection with robot and it can be given capability to complete the performance operation with high accuracy.The proposed robot used PLC for controlling the system runs faster, with high efficiency , without error where the desired trajectory equal the actual path because the selected angles which were obtained from inverse kinematic identifiy in the PLC then the running process gave the same position to the simulate path which hade draw in Matlab. Moreover PLC has a short production cycle and it can be obtain a new trajectory motion by only changing the program of (Zelio PLC).

The implemented SCARA robot can be used for welding processes and other applications like pick and place, cutting and drilling by just replacing the tool.

\section{References}

[1] MarkW.S,Seth H. ,andMathukumalli V., "Robot Dynamics and Control" ,book $2^{\text {nd }}$, John Wiley \& Sons, Inc. 2008.

[2] Viliam F.,František Ď.,andRóbertÜ.,"Analysis of Robotic System MotioninSimMechanicsandMATLAB GUI Environment" INTECH, 2014.

[3] ALAVUDEEN A., VENKATESHWARAN N., "Computer Integrated Manufacturing ", PHI Learning Private Limited 2010.

[4] William B., "Programmable logic controls", book $4^{\text {th }}$ edition, Newnes 2006.

[5] PouyaJ.andKouroshH.S.,"RobotManipulators: Modeling, SimulationandOptimalMultiVariable Control,"Appl. Mech. Mater., vol.232, pp.383-387, 2012.

[6] MohammadA. andMahdiS.A.,"SCARArobotcontrol usingneuralnetworks, 'ICIAS,vol.1, pp.126130, 2012.

[7]FirasA. T.andAhmedA. A.,"Implementationof FPGA BasedPSOPIDController for FeedbackIVAXSCARARobot Manipulator", IJCSET vol.3,no.10,pp.367-372,2013.

[8] Jian F.and Wei L.,"Fourdegreesof freedomSCARArobot kinematics modeling and simulation analysis SCARA Robot Kinematics”,IJ3C,vol.2,no.4, 2013.

[9] SudhaR.,RaghavV.S.,ArkoC.,andand PadmaT., "DEVELOPMENT $\mathrm{OF}$ SCARAMANIPULATORWITHTWO DEGREES OFFREEDOM USINGDC MOTOR,",ARPN Journal,vol.8,no.6,pp.445448, 2013.

[10] YousifA.M.,"Design,Analysis,Simulation, an dVirtual RealityVerifiedIntelligentController forIndustrialApplication SCARARobot," ,International Journalof Advanced Computing ,vol.46,no.3, pp. 1316-1325, 2013.

[11] ZelunL.,ZhichengH.,and YoujunH.,"Designof Spot WeldingRobot," ,Telkomnika Indonesian Journalvol. 11, no. 11, pp. 62676273,2013.

[12] PanchanandJ.and Biswal B.B.,"ANeural Network Approach for Inverse Kinematicof a SCARA Manipulator,"IJRA, vol.3, no.1, pp. 52-61, 2014.

[13] M.Shariatee, A.Akbarzadeh, A.Mousavi,andS.Alimardani, "Designof anEconomicalSCARARobotfor Industrial Applications,"pp. 534-539,2014. 
[14] NiteshK.J.andVijayK.,"3-DOF Scara type RobotManipulator usingMamdaniBasedFuzzyController",IJSRE Tvol.3,no.3, pp. 659-663, 2014.

[15] Subhashini P. V. S. ,, RajuN.V.S. and Rao G. V."Modeling,SimulationandAnalysisof a ScaraRobot,"ARPN J.Eng. Appl.Sci., vol.9, no.4, pp. 398-404, 2014.

[16] Pouya J.and Kourosh H.S.,"Robot Manipulators:Modeling, Simulation and Optimal Multi-Variable Control,"Appl. Mech. Mater., vol.232, pp.383-387, 2012.

[17] https://www.robots.com/faq/show/what-arethe-maintypes-of-robots.
[18] [18] Kharagpur, "Programming of PLCs: Sequcutial Function Charts", Version 2 EE IIT.

[19] Ted Thayer, "Speaking in Tongues: Understanding the IEC 61131-3 Programming Languages",Bosch Rexroth Electric Drives and Controls 2009, Plant Engineering Provides. 
تصميم وتنفيذ روبوت نوع سكارا ثلاثي درجات الحرية معتمدا مسيطر المنطق المبرمج

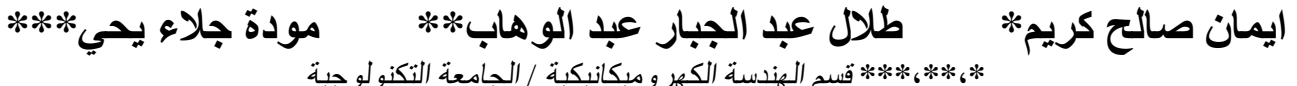

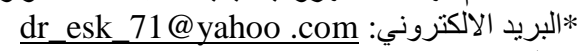

$$
\begin{aligned}
& \text { electromechanical19@yahoo.com البريد الالكترونيد البكروني:**** }
\end{aligned}
$$

يقدم البحث الحالي عملية التصميم و التنفيذ الميكانيكي و الكهربائي لروبوت صناعي نوع SCARA ثلاثي درجات الحرية ذي حركتين دور انيه افقية،

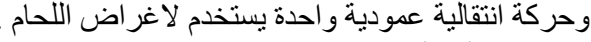

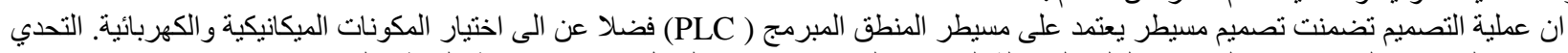

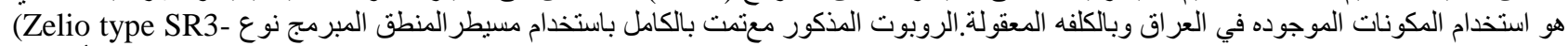

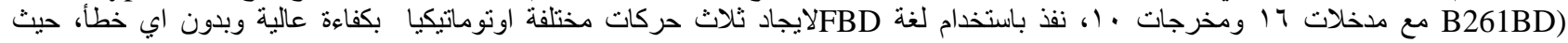

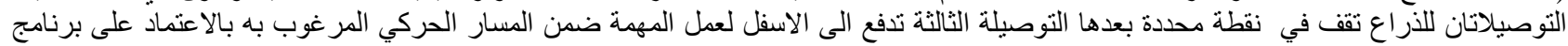

